\title{
Chemistry of Ammonium Betaines: Application to Ion-Pair Catalysis for Selective Organic Transformations
}

\author{
Daisuke Uraguchi ${ }^{1}$ and Takashi Ooi ${ }^{1,2 *}$ \\ ${ }^{1 *}$ Institute of Transformative Bio-Molecules (WPI-ITbM) and Department of Molecular and \\ Macromolecular Chemistry, Graduate School of Engineering, Nagoya University \\ Nagoya 464-8601, Japan \\ 2* CREST, Japan Science and Technology Agency (JST), Nagoya University \\ Nagoya 464-8601, Japan
}

(Received August 10, 2018; E-mail: tooi@chembio.nagoya-u.ac.jp)

\begin{abstract}
The ammonium betaine was developed as an intramolecular ion-pair catalyst for realizing cooperative catalysis of a cation and anion; i.e. ion-pair catalysis. Combination of the stereocontrolling ability of the chiral ammonium ion and functions of the pairing aryloxylate ion enabled bifunctional organic base catalysis and ionic nucleophilic catalysis, which facilitate a variety of organic transformations with rigorous stereochemical control. In addition, employing a single-electron-accepting cation as a partner of the basic aryloxylate led to the development of a chemical redox catalyst with the capability of proton-coupled electron transfer (PCET). This research demonstrates a power of the ion-pair catalysis in selective organic synthesis and is therefore likely to stimulate further study in this field.
\end{abstract}

\section{Introduction}

Since a landmark thesis of the Merck research group in 1984 disclosed that cinchona alkaloid-derived chiral ammonium salt could precisely dictate the absolute stereochemistry of alkylation of a prochiral enolate under aqueous-organic biphasic conditions, ${ }^{1}$ the chemistry of chiral quaternary onium salts has been placed at the central position in the asymmetric counterion-directed catalysis. ${ }^{2}$ However, the salt has been mainly utilized for phase-transfer catalysis and thus, the potential of its catalytic performance has not yet been fully explored, especially under homogeneous conditions. ${ }^{3}$ This is largely because of difficulties associated with the understanding of the reaction mechanism that involves dynamic ion exchange under biphasic conditions; that has hampered the application of chiral quaternary onium salt catalysis to a variety of organic transformations in non-specific media.

In the general mode of chiral quaternary onium salt catalysis, the chiral onium ion exchanges its pairing anion with a reactive anion such as an enolate to form the corresponding chiral ion pair, and the ion-paired chiral intermediate participates in downstream bond formation with control of the stereochemical outcome. Accordingly, the cation is a key component responsible for asymmetric catalysis, while the parent anion of the catalyst hardly contributes to the overall reaction, other than to the efficiency of the initial ion-exchange step. In this regard, the catalysis should be classified as chiral cationdirected catalysis, and the development of cooperative catalysis of a cation and anion (ion-pair catalysis) remained elusive, despite its significant potential for expanding the scope of counterion-directed catalysis. ${ }^{2}$ This situation led us to embark on the development a strategy for establishing true ion-pair catalysis. The simplest approach to this direction was to fix an anion near to a cation by connecting two ions with a covalent bond to form an intramolecular ion pair; thus, we became interested in a betaine. ${ }^{4}$

Betaine is historically reserved for trimethyglycine, but nowadays it is defined as a zwitterionic molecule with a nonprotonated cation and any anionic site at non-adjacent positions in a single molecule. ${ }^{5}$ Since the two ionic sites in the betaine are embedded in a spacer scaffold, the structure of the ion pair can be directly regulated through modification of the spacer skeleton, allowing the generation of a structured ion pair. We envisaged that judicious use of the characteristic features of betaine would allow us to design the entire structure of the ion pair for catalytic transformations enabled by the simultaneous function of a cation and anion, thereby opening a door to the ion-pair catalysis. In this account, we describe our efforts toward the development of ion-pair catalysis using ammonium betaines.

\section{Bifunctional Organic Base Catalysis}

If the anion moiety of the betaine abstracts a proton from a pronucleophile as a Brønsted base, the resulting conjugate acid would remain as a hydrogen-bond donating site at a neighboring position to the cationic center and could participate in capturing the nucleophilic anion in cooperation with ionic interaction to form a structured ion pair (Figure 1, lower panel). This contrasts markedly to the conventional intermolecular ion pair, where the parent anion is liberated from the reactive yet fluxional ion pair after the proton transfer event (Figure 1, upper panel). ${ }^{6}$ We envisioned that formation of the structured ion pair could help us design stereoselective catalyst systems through an understanding of the reaction mechanism. Furthermore, the cooperative operation of electrostatic attraction by the ammonium ion and the dual function of the anion moiety could render the betaine a bifunctional organic base catalyst. To assess the validity of this hypothesis, we designed an axially chiral ammonium betaine of type 1 possessing aryloxy moiety as a basic anionic functionality (Figure 2a). ${ }^{7,8}$ The structural characteristics of betaine $\mathbf{1}$ are as follows: (1) the axially chiral binaphthyl backbone could flexibly adjust the distance of the ammonium ion and aryloxylate; (2) the substituent at the ortho-position of aryloxylate (Ar) would pro- 


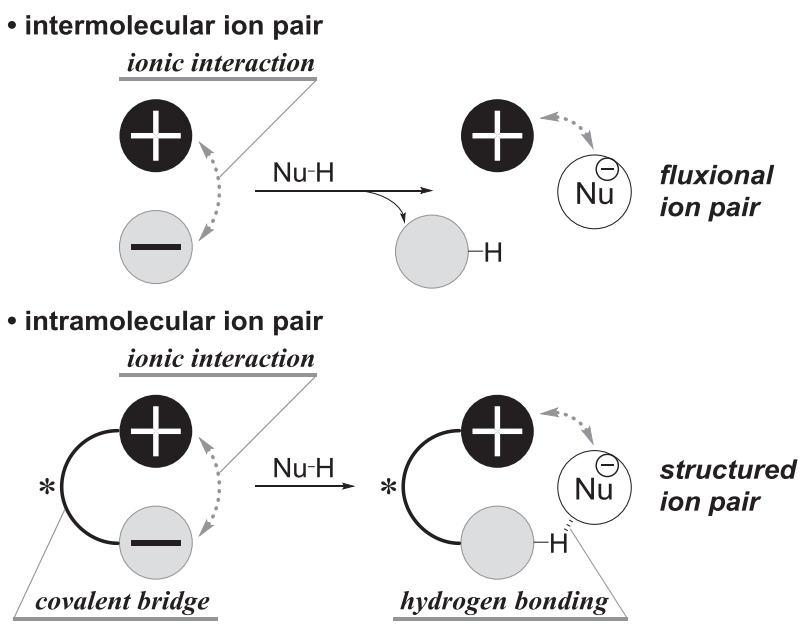

Figure 1. Inter- vs. intra-molecular ion-pair catalysis with a basic anionic component.

vide an effective chiral environment around the reaction sphere; (3) the ortho-substituent of the other naphthyl unit (R) could restrict the rotation of the ammonium appendage at the benzylic carbon; and (4) the intramolecular ionic interaction would regulate the direction of the cationic site, which could eventually control the position of the nucleophilic anion (see Figure 1).
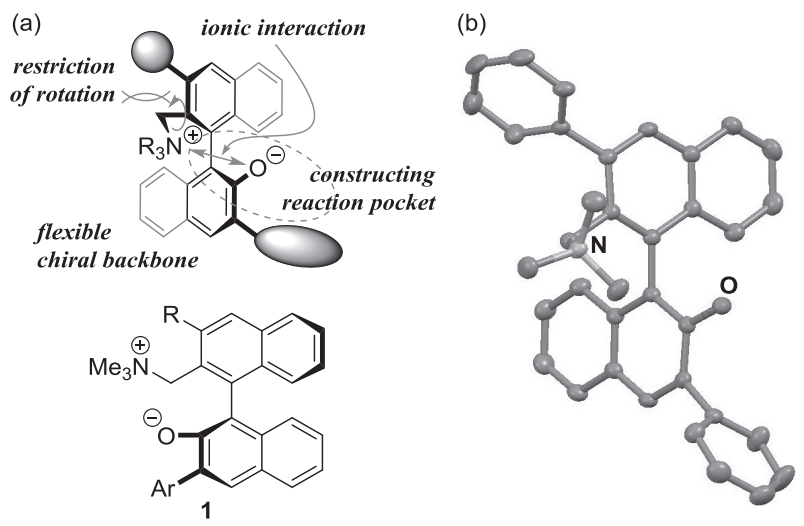

Figure 2. (a) General structure of axially chiral ammonium betaines 1. (b) ORTEP diagram of $\mathbf{1 b}(\mathrm{Ar}, \mathrm{R}=\mathrm{Ph})$. Calculated hydrogen atoms and solvent molecules $\left(\mathrm{MeOH}\right.$ and $\left.\mathrm{H}_{2} \mathrm{O}\right)$ are omitted for clarity.

First, we pursued establishment of a reliable procedure for the formation of the requisite intramolecular ion pair. ${ }^{7}$ Chiral ammonium betaines $\mathbf{1}$ were quantitatively prepared as a bench stable yellowish solid from its precursor, the quaternary ammonium trifluoroacetate $\mathbf{1} \cdot \mathrm{HOCOCF}_{3},{ }^{9}$ by a simple treatment with a $0.1 \mathrm{M}$ aqueous solution of sodium bicarbonate except for the simplest betaine 1a $(\mathrm{R}, \mathrm{Ar}=\mathrm{H})$. Since 1a is a highly water-soluble molecule, a non-aqueous procedure; i.e. treatment of 1a with anhydrous tripotassium phosphate in acetone, was required for preparative isolation. Single crystal $\mathrm{X}$-ray diffraction analysis of $\mathbf{1 b}(\mathrm{R}, \mathrm{Ar}=\mathrm{Ph})$ revealed its three dimensional structure, where the trimethylammonium cation moiety turned in the same direction as the aryloxide oxygen to form the expected intramolecular ion pair $(\mathrm{N} \cdots \mathrm{O}=4.271 \AA)$ (Figure 2b).

\subsection{Mannich-type Reaction of $\alpha$-Nitro Carboxylates}

As a starting point of exploring betaine catalysis, we chose the Mannich-type reaction of $\alpha$-nitro carboxylate with $N$ Boc aldimine for evaluating the catalytic performance of $\mathbf{1}^{7}$ Fortunately, the simplest betaine 1a exhibited an ability to catalyze the Mannich-type reaction at $0{ }^{\circ} \mathrm{C}$ in toluene and the adduct was isolated in $40 \%$ yield after $20 \mathrm{~h}$ of stirring (Table 1 , entry 1). However, the stereoselectivity of the reaction was rather poor. As expected, introduction of substituents to the 3,3 -position of the binaphthyl backbone greatly improved the catalytic activity and stereocontrolling ability, and 3,3'-phenyl-substituted $\mathbf{1 b}$ gave a diastereomeric mixture of the adduct (syn/anti $=2.1: 1$ ) in $92 \%$ yield with $90 \%$ ee for the $s y n$-isomer (entry 2). Changing the substituent at the ortho-position to the ammonium moiety from phenyl to chlorine slightly enhanced the stereoselectivity (entry 3 ). With respect to the ortho-aromatic substituent of aryloxylate, increasing steric bulkiness in the para-direction was beneficial, but similar steric demand in the meta-direction diminished stereoselectivity (entries 4 and 5). While 1 consisting of a single binaphthyl backbone displayed excellent enantioselectivity, expanding its molecular structure to a pseudo- $C_{2}$ symmetric form (Figure 3) was found

Table 1. Structure-selectivity relationship of the chiral ammonium betaine $\mathbf{1}$ and $\mathbf{2}$ in the Mannich-type reaction.

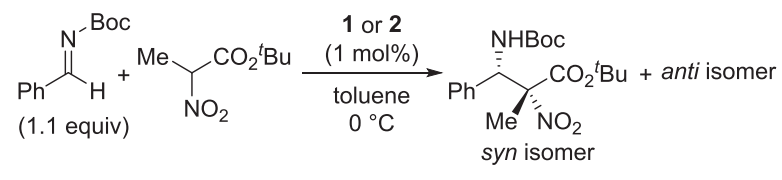

\begin{tabular}{cccccc}
\hline entry & $\begin{array}{c}\text { catalyst } \\
\text { or } \mathbf{2}(\mathrm{R}, \mathrm{Ar})\end{array}$ & $\begin{array}{c}\text { time } \\
(\mathrm{h})\end{array}$ & $\begin{array}{c}\text { yield } \\
(\%)\end{array}$ & $\begin{array}{c}\mathrm{dr} \\
(\text { syn/anti })\end{array}$ & $\begin{array}{c}\text { ee } \\
(\%)\end{array}$ \\
\hline $1^{a}$ & $\mathbf{1 a}(\mathrm{H}, \mathrm{H})$ & 20 & 40 & $1: 1.2$ & $12 / 18$ \\
2 & $\mathbf{1 b}(\mathrm{Ph}, \mathrm{Ph})$ & 10 & 92 & $2.1: 1$ & 90 \\
3 & $\mathbf{1 c}(\mathrm{Cl}, \mathrm{Ph})$ & 8 & 89 & $2.6: 1$ & 93 \\
4 & $\mathbf{1 d}\left(\mathrm{Cl}, 4-{ }^{t} \mathrm{BuC} \mathrm{H}_{4}\right)$ & 8 & 98 & $3.2: 1$ & 95 \\
5 & $\mathbf{1 e}\left(\mathrm{Cl}, 3,5-{ }^{t} \mathrm{Bu}_{2} \mathrm{C}_{6} \mathrm{H}_{3}\right)$ & 36 & 91 & $1.6: 1$ & 47 \\
$6^{a}$ & $\mathbf{2 a}(\mathrm{H}, \mathrm{H})$ & 10 & 93 & $1: 1$ & $-24 / 16$ \\
$7^{a}$ & $\mathbf{2 b}(\mathrm{H}, \mathrm{Ph})$ & 8.5 & 93 & $1: 1$ & $98 / 95$ \\
$8^{a}$ & $\mathbf{2 c}(\mathrm{Me}, \mathrm{Ph})$ & 2 & 91 & $2.0: 1$ & 99 \\
$9^{a}$ & $\mathbf{2 d}(\mathrm{Cl}, \mathrm{Ph})$ & 5 & 95 & $4.1: 1$ & 99 \\
10 & $\mathbf{2 d}$ & 8 & 97 & $3.9: 1$ & 99 \\
$11^{a}$ & $\mathbf{2 d}$ & 5 & 97 & $1.3: 1$ & $-5 / 5$ \\
\hline${ }^{a} 5$ mol\% of the catalyst was used. & & & &
\end{tabular}

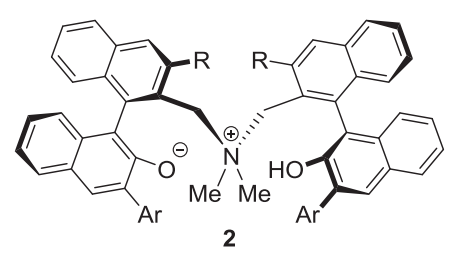

2a: $\mathrm{R}=\mathrm{Ar}=\mathrm{H} \quad$ 2c: $\mathrm{R}=\mathrm{Me}, \mathrm{Ar}=\mathrm{Ph}$ 2b: $\mathrm{R}=\mathrm{H}, \mathrm{Ar}=\mathrm{Ph} \quad$ 2d: $\mathrm{R}=\mathrm{Cl}, \mathrm{Ar}=\mathrm{Ph}$

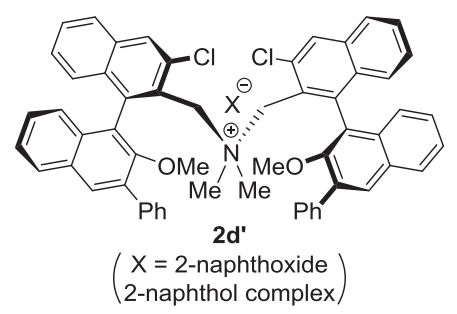

Figure 3. Structure of pseudo- $C_{2}$ symmetric chiral ammonium betaines $\mathbf{2}$ and intermolecular analog $\mathbf{2 d}$ '. 
to be effective for further improving enantioselectivity. Among the betaines tested, 2d showed highest diastereoselectivity and virtually complete enantioselectivity (entries 6-10). It is noteworthy that the intermolecular-type ammonium aryloxylate 2d' provided a nearly racemic adduct, although the reaction efficiency was retained (entry 11), which clearly indicated the importance of the intramolecular ion-pair nature of the ammonium betaine $\mathbf{2}$ in achieving the absolute stereocontrol.

The catalysis was applicable to a wide variety of $N$-Boc aldimines and high yield, moderate diastereoselectivity, and excellent enantioselectivity were generally observed (Scheme 1).

Scheme 1. Chiral ammonium betaine 2d-catalyzed Mannich-type reaction of $\alpha$-nitro carboxylate.

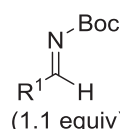

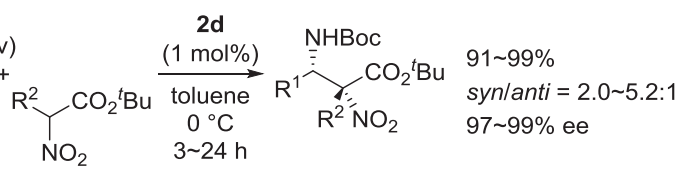

\subsection{Mannich-type Reaction of 2-Alkoxythiazol-5(4H)-ones}

The highly enantioenriched product in the above reaction can serve as a precursor of $\alpha, \beta$-diamino acids, yet its diastereoselectivity is not synthetically useful. Therefore, we turned our attention to apply the catalysis to another Mannich-type reaction that is more suitable for the preparation of $\alpha, \beta-$ diamino acids. ${ }^{10}$ For this purpose, we selected 2-alkoxythiazol$5(4 H)$-one (thiazolone) as a nucleophilic component with expectation that its rigid structure would be advantageous for precisely dictating the stereochemistry of the bond formation. ${ }^{11}$ In addition, we assumed that thiazolone could be converted into $N$-protected $\alpha$-amino acid in a facile manner, in contrast to its oxygen-analog, oxazol-5(4H)-one (azlactone). ${ }^{12}$ The investigation of the appropriate reaction conditions made it clear that the bifunctional organic base catalysis of betaine 1 was indeed effective for the Mannich-type reaction of thiazolones with $N$-Boc aldimines. ${ }^{8}$ In this reaction, the structure of the 3-substituent on the naphthyl unit of the aryloxylate side was key for rigorously controlling the stereochemical outcome. Extensive survey of the structure-selectivity relationship revealed that betaine 1f possessing an extremely bulky aromatic group was the best catalyst for the present Mannich-type reaction (Scheme 2).

Scheme 2. Chiral ammonium betaine 1f-catalyzed Mannich-type reaction of thiazolones.

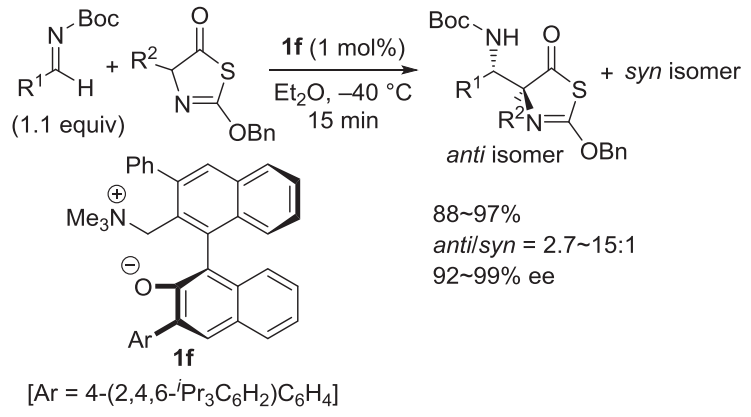

With the stereochemically enriched Mannich adduct in hand, we attempted to establish its derivatization into $\alpha, \beta$ diamino acid derivatives to demonstrate the synthetic utility of the 1f-catalyzed Mannich protocol (Scheme 3). The thiazolone ring was easily opened upon treatment with basic hydrogen peroxide, and concomitant oxidation of the resulting thiocarbonyl moiety into benzyloxycarbonyl afforded differently protected $\alpha, \beta$-diamino acid in good yield. Furthermore, we found that the benzyl group could be removed by exposure to methanolic hydrogen chloride to produce 4 -tetrasubstituted thiazolidine-2,5-dione. The subsequent aminolysis of the thiazolidine-2,5-dione ring with methylamine furnished an $\alpha, \beta-$ diamino amide, featuring the reactivity of the thiazolidine2,5-dione as an acyl-transfer reagent.

Scheme 3. Synthesis of various $\alpha, \beta$-diamino acid derivatives from the Mannich adduct of thiazolone.

$$
\text { (10:1,99\% ee) }
$$

\subsection{Mannich-type Reaction of 3-Aryl Oxindoles}

A betaine catalyst is also effective for controlling the stereochemistry of the Mannich-type reaction of 3-aryl oxindoles (Scheme 4). ${ }^{13}$ Despite the importance of 3-quaternary chiral oxindoles in the synthesis of biologically relevant molecules, examples of catalytic asymmetric Mannich-type reactions of oxindoles are rare and 3-aryl oxindoles are regarded as a particularly problematic substrate. ${ }^{14,15}$ We solved this problem by utilizing bifunctional organic base catalysis of betaine 1 . In fact, the catalytic amount of betaine $1 \mathrm{~g}$ having $4-$ tert-butylphenyl groups at 3,3'-positions of the binaphthyl backbone promoted smooth Mannich-type reaction and provided the corresponding adducts in high yield with excellent stereoselectivity.

Scheme 4. Chiral ammonium betaine 1g-catalyzed Mannich-type reaction of 3-aryl oxindoles.
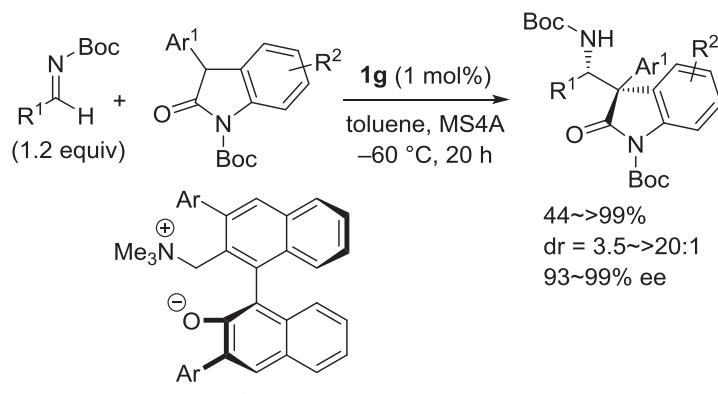

$44 \sim>99 \%$

$\mathrm{dr}=3.5 \sim 20: 1$

93 $99 \%$ ee

$1 \mathrm{~g}\left(\mathrm{Ar}=4-{ }^{\mathrm{t}} \mathrm{BuC}_{6} \mathrm{H}_{4}\right)$

\subsection{Mannich-type Reaction of Dihydro-2(1H)-quinolones}

The high performance of betaine in the Mannich-type reactions of oxindoles led us to become interested in using dihydro-2(1H)-quinolones as a pronucleophile. ${ }^{16}$ Although various annulation reactions have been introduced to access synthetically valuable chiral dihydroquinolone derivatives, asymmetric modification of their lactam ring remained a chal- 
lenge. ${ }^{17}$ We thus employed 3-nitro-dihydro-2(1H)-quinolone as a nucleophilic component of the Mannich-type reaction under the catalysis of chiral ammonium betaine 1. After optimization of the catalyst structure and reaction conditions, a fairly stereoselective system was established with $\mathbf{1 h}$ as a requisite catalyst (Scheme 5).

Scheme 5. Chiral ammonium betaine $\mathbf{1 h}$-catalyzed Mannich-type reaction of dihydro-2(1H)-quinolones.

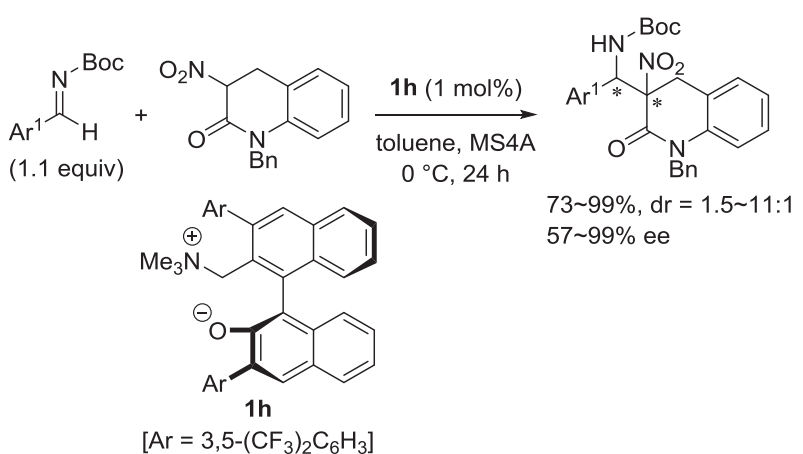

\subsection{Aza-Henry Reaction of Vinylogous Nitronates of $\beta, \beta$-Disubstituted Nitroolefins}

With the prominent catalytic function of the betaine in mind, we next turned our attention to exploring a novel nucleophilic component. In this regard, we were interested in a vinylogous nitronate. Since the nitro group is a valuable functionality that can be readily converted into amino and carbonyl groups, catalytic asymmetric transformations of nitro-containing compounds have been extensively studied. ${ }^{18}$ Nevertheless, the chemistry of vinylogous nitronates is poorly understood, in marked contrast to the rich chemistry of vinylogous enolates. ${ }^{19}$ Considering the high acidity of the $\alpha-$ proton of nitroalkanes, the $\gamma$-proton of conjugated nitroolefins could be easily deprotonated in principle; however, generation of the vinylogous nitronate has never been investigated under catalytic conditions. Therefore, feasibility of the catalytic generation of vinylogous nitronates and their synthetic applicability, including the intrinsic regiochemical preference ( $\alpha$ vs. $\gamma$-addition), remained unclear. Under these circumstances, previous reports on the methodology development with vinylogous enolates generated from $\alpha, \beta$-unsaturated carbonyl compounds gave us a hint that the doubly $\beta$-substituted structure would be a prerequisite for the facile generation of vinylogous enolates. We reasoned that steric repulsion between one of the $\beta$-substituents and an electron-withdrawing group, such as a carbonyl component, facilitates deprotonation at $\gamma$ position due to relaxation of the repulsion upon formation of the corresponding vinylogous enolate. If this mechanism is operative with $\beta, \beta$-disubstituted nitroolefins, facile $\gamma$-deprotonation is conceivable, providing an opportunity to exploit the vinylogous nitronate as a nucleophile (Figure 4).

As expected, treatment of $\beta$-methyl nitrostyrene with $N$ Boc benzaldimine in the presence of ammonium betaine $\mathbf{1 b}$ in toluene at $-30{ }^{\circ} \mathrm{C}$ gave rise to the $\alpha$-isomer of the aza-Henry adduct as a single diastereomer in $77 \%$ yield with $97 \%$ ee. ${ }^{20}$ The catalytic efficiency and stereoselectivity were further improved by changing the aromatic substituents of betaine and 1i possessing 4-trifluoromethyphenyl groups on both 3-positions was the optimal catalyst for obtaining a product in excellent yield with virtually complete stereoselectivity (Scheme 6).

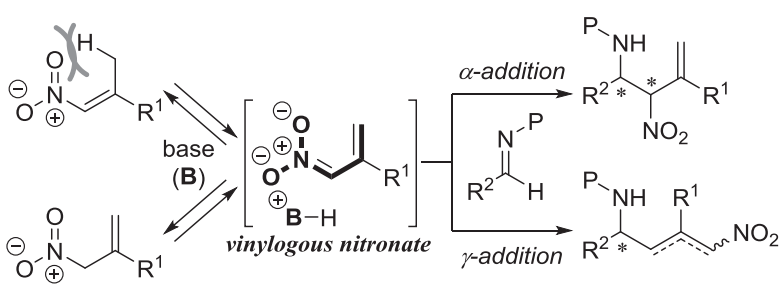

Figure 4. Generation of vinylogous nitronates from $\beta, \beta$-disubstituted nitroolefins.

Importantly, no conversion was confirmed when subjecting a $\beta$-mono-substituted nitroolefin to the reaction conditions, proving that two $\beta$-substituents were essential in this azaHenry reaction. It is worth noting that the corresponding $\gamma$ isomer was never detected, which suggests the intrinsic $\alpha$ preference of the vinylogous nitronate during carbon-carbon bond formation with $N$-Boc aldimines.

Scheme 6. Chiral ammonium betaine 1i-catalyzed aza-Henry reaction of $\beta, \beta$-disubstituted nitroolefins.

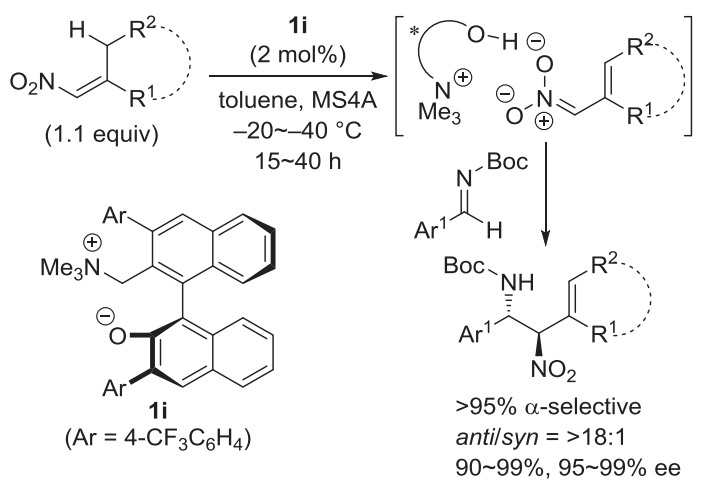

\subsection{Aza-Henry Reaction of Vinylogous Nitronates of $\alpha$-Aryl $\beta$-Substituted Nitroolefins}

The importance of steric repulsion for the facile generation of vinylogous nitronates led us to explore another substitution pattern of nitroolefins pertinent to accelerating $\gamma$-deprotonation. We hypothesized that introduction of an aromatic substituent to $\alpha$-position might induce beneficial 1,3-allylic repulsion between a $\beta$-substituent and a nitro group as illustrated in Figure 5. ${ }^{21}$ Indeed, $\alpha$-aryl $\beta$-mono-substituted nitroolefins underwent facile $\gamma$-deprotonation by the action of betaine 1 to generate the corresponding vinylogous nitronate that was amenable to the stereoselective aza-Henry reaction with $N$-Boc aldimines. Optimization of the betaine structure and reaction conditions allowed us to selectively obtain the $\alpha-$ adduct in high yield with excellent stereoselectivity under the

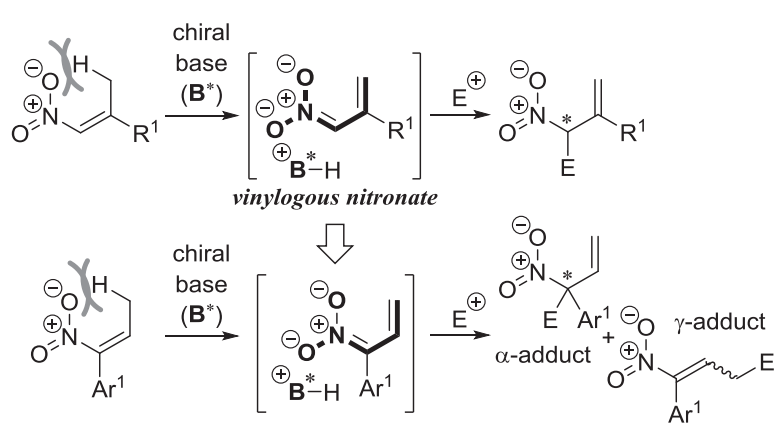

Figure 5. Generation of vinylogous nitronate from $\alpha$-aryl $\beta$-mono-substituted nitroolefin. 
influence of $\mathbf{1 h}$ (Scheme 7). Here again, $\gamma$-adduct formation was not detected and the bond formation at the sterically congested $\alpha$-position underscored the strong preference of the vinylogous nitronate toward $\alpha$-addition.

Scheme 7. Chiral ammonium betaine $\mathbf{1 h}$-catalyzed Henry reaction of $\alpha$-aryl $\beta$-mono-substituted nitroolefins.

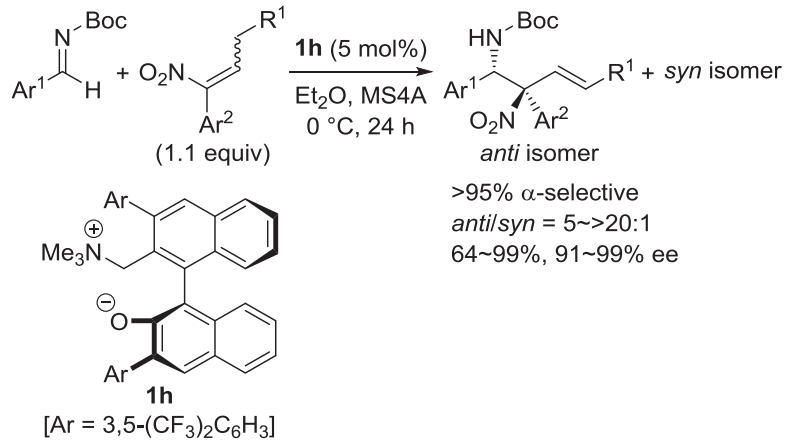

\subsection{Aza-Henry Reaction of $\alpha$-Aryl Nitromethanes}

The betaine 1-catalyzed aza-Henry protocol was applicable to $\alpha$-aryl nitromethanes in a similar manner, providing an access to a valuable precursor of 1,2-diaryl ethylenediamines (Scheme 8). ${ }^{22,23}$ High stereoselectivity was accomplished by using ammonium betaine $\mathbf{1 i}$ as a catalyst. The aza-Henry adduct thus obtained was easily converted into the corresponding $N$-unprotected diamine without loss of stereochemical integrity, demonstrating its synthetic utility. Notably, when the product was treated with triethylamine, facile epimerization at the stereogenic carbon attached to the nitro group took place, resulting in a mixture of a nearly equal amount of diastereomers, while the enantiomeric excesses of both diastereomers remained intact. This observation suggests that the betaine catalyst rigorously discriminates the prochiral faces of $N$-Boc aldimines at the carbon-carbon bond-forming event.

Scheme 8. Chiral ammonium betaine 1i-catalyzed Henry reaction of $\alpha$-aryl nitromethanes.

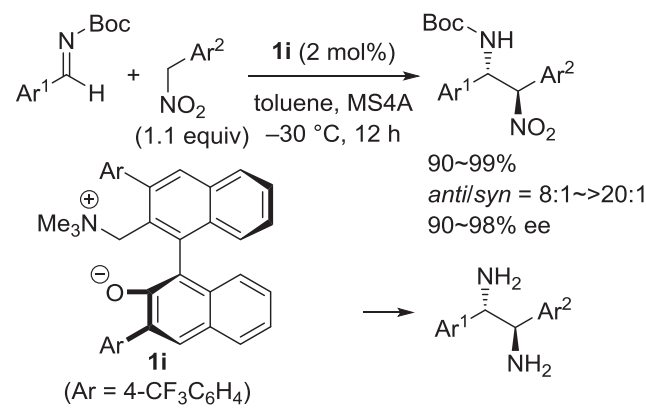

\section{Ionic Nucleophilic Catalysis}

In addition to the basic character, aryloxylate functionality of ammonium betaine also exhibits nucleophilicity. ${ }^{6}$ This attribute inspired us to conceive that betaine 1 could be evolved into a chiral nucleophilic catalyst through appropriate structural manipulations, despite the fact that most, if not all, of the previously reported nucleophilic catalysts were non-ionic molecules and that development of an ionic nucleophilic catalyst has been elusive. ${ }^{24}$

The nucleophilic catalyst is unique in temporally forming a covalent bond with the substrate to produce a reactive inter- mediate, which is eventually incorporated in the product. For instance, in the acyl transfer reaction to an anionic substrate, a conventional, non-ionic nucleophilic catalyst attacks an acylating reagent to generate the ion pair, and ion exchange followed by bond formation affords the product (Figure 6a). In contrast, if the catalyst is an ionic molecule such as onium aryloxylate $\left(\mathrm{Q}^{+} \mathrm{OAr} \mathrm{r}^{--}\right)$, the catalyst loses its charge upon reacting with the acylating reagent, and the resulting non-ionic intermediate ( $\mathrm{R}^{\prime} \mathrm{COOAr}$ ') is less reactive than the ionic one (Figure 6b). This is presumably the most critical disadvantage and ground for the lack of ionic nucleophilic catalysts. This understanding led us to apply the betaine as a nucleophilic catalyst. When a betaine molecule is employed as an ionic catalyst to the acyl transfer reaction, the problematic non-ionic intermediate possesses an anchimeric cationic moiety; thus, after ion exchange, the final bond formation should proceed pseudo-intramolecularly, thereby circumventing the inherent reactivity impediment (Figure 6c). In addition, we anticipated that the unique intermediary ion pair could also induce an unprecedented level of stereocontrol. (a) non-ionic catalysis

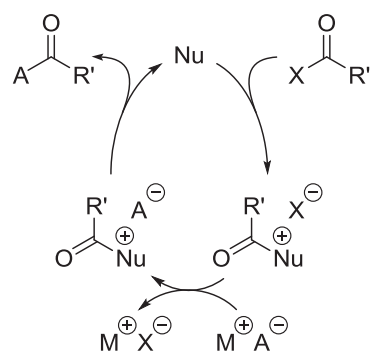

(b) intermolecular ionic catalysis

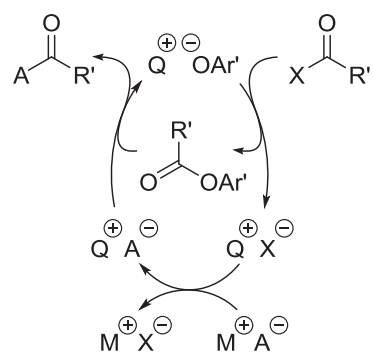

(c) intramolecular ionic (betaine) catalysis

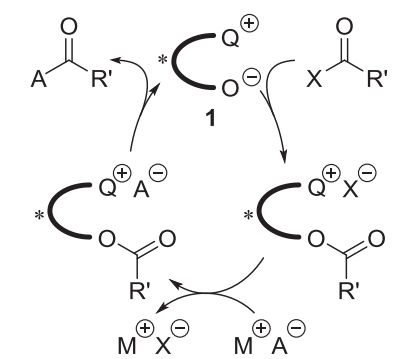

Figure 6. Mode of nucleophilic catalysis in the acyl transfer reaction to an ionic reagent.

\subsection{Steglich Rearrangement}

Among acyl transfer reactions under nucleophilic catalysis, the formal rearrangement of 5-oxazolyl carbonate into 4-carboxyazlactones (the Steglich rearrangement) has served as a model system for evaluating the capability of chiral nucleophilic catalysts. ${ }^{25}$ Therefore, we examined the performance of betaine 1 as an ionic nucleophilic catalyst in the Steglich rearrangement of 2-tert-butyl-4-benzyl-5-oxazolyl 2,2,2-trichloroethyl carbonate and found that $\mathbf{1 b}$ could not promote the desired reaction (Scheme 9). ${ }^{26}$ We inferred that the steric hindrance of the 3-aryl group of the aryloxylate side hampered the acyl transfer process and thus prepared less hindered betaine $1 \mathbf{j}$ by removing the aryl group. ${ }^{27}$ When the phenylalanine-derived carbonate was added dropwise to a stirred mixture of betaine $1 \mathbf{j}(2 \mathrm{~mol} \%)$ and powdered $4 \AA$ molecular sieves in 1,4-dioxane at $25^{\circ} \mathrm{C}, 4$-alkoxycarbonylazlactone was smoothly produced in $93 \%$ yield with $93 \%$ ee. It was of interest 
that the characteristic yellow color of $\mathbf{1 j}$ immediately lightened upon the addition of one drop of a solution of the carbonate, which was an indication of acylation of the aryloxylate moiety of $\mathbf{1} \mathbf{j}$ to form the ammonium enolate. After leaving the mixture for a while without further addition of the carbonate, the original yellow color gradually returned, indicating the regeneration of $\mathbf{1} \mathbf{j}$ with concomitant formation of the product. This observation suggests that carbon-carbon bond formation is the rate-limiting step in this catalysis. The introduction of an electron-withdrawing group such as 4-trifluoromethylphenyl (1k) or 3,5-bis(trifluoromethyl)phenyl (11) to the naphthyl unit bearing the ammonium cation enhanced catalytic efficiency, as indicated by the vivid yellow color remaining throughout the reaction, and improved enantioselectivity further to $95 \%$ ee and $97 \%$ ee, respectively.

Scheme 9. Evaluation of the performance of ammonium betaine $\mathbf{1}$ as an ionic nucleophilic catalyst.

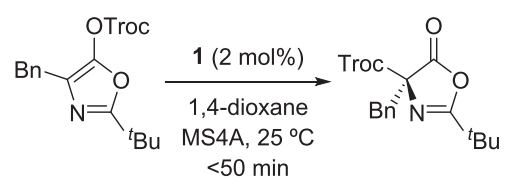

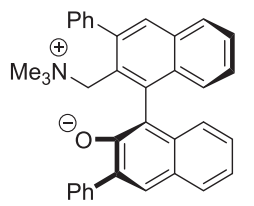

1b: no reaction

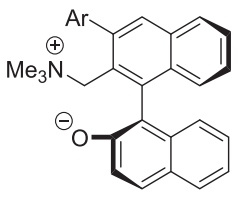

$1 \mathrm{j}(\mathrm{Ar}=\mathrm{Ph}): 93 \%, 93 \%$ ee $1 \mathbf{k}\left(\mathrm{Ar}=4-\mathrm{CF}_{3} \mathrm{C}_{6} \mathrm{H}_{4}\right): 96 \%, 95 \%$ ee 1I $\left[\mathrm{Ar}=3,5-\left(\mathrm{CF}_{3}\right)_{2} \mathrm{C}_{6} \mathrm{H}_{3}\right]: 97 \%, 97 \%$ ee
The optimized betaine $\mathbf{1 l}$ exhibited extremely high catalytic performance and a series of carbonates was converted into the corresponding azlactones within $30 \mathrm{~min}$ quantitatively with excellent enantiomeric excesses (Scheme 10). Even sterically hindered carbonate $\left(\mathrm{R}^{1}={ }^{i} \mathrm{Pr}\right)$ underwent rearrangement by raising the temperature to $40{ }^{\circ} \mathrm{C}$ to afford the product with $95 \%$ ee, implying that the prominent nucleophilicity of the anion part and unique structure of the betaine play crucial roles in the nucleophilic catalysis.

Scheme 10. Chiral ammonium betaine 11-catalyzed enantioselective Steglich rearrangement.
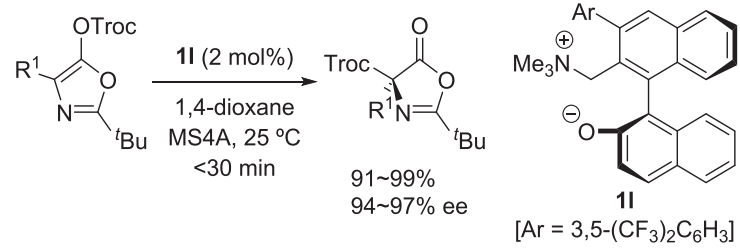

An interesting aspect of the present reaction system is the dependence of enantioselectivity on substrate concentration, and low concentration of the carbonate appeared to be key for attaining high enantiomeric excess. In fact, enantioselectivity of the reaction decreased from $97 \%$ to $87 \%$ when $\mathbf{1 l}$ was added to a solution of the phenylalanine-derived carbonate under otherwise identical conditions. This outcome suggests that two reaction pathways exist in the 1-catalyzed Steglich rearrangement. One is the expected pseudo-intramolecular "rearrangement" with high enantioselectivity (Figure 6a, cycle I) and the other is the less stereoselective intermolecular reaction of the ammonium enolate with the external carbonate (cycle II). The possible influence of this undesired intermolecular pathway on the reaction profile was assessed by investigating the reactivity of the carbonate as an acyl-transfer reagent to an ammonium enolate. Namely, the addition of the carbonate to a solution of in situ generated tetrabutylammonium $\beta$-naphthoxide (20 $\mathrm{mol} \%$ ) produced azlactone in $85 \%$ yield along with $2,2,2-$ trichloroethyl $\beta$-naphthyl carbonate in $15 \%$ yield (Figure $6 \mathrm{~b}$ ). This result indicates that the intermediary ammonium enolate, which was generated via initial nucleophilic addition of $\beta$ naphthoxide to the carbonate, directly reacted with the carbonate to give azlactone. In other words, the chiral ammonium enolate could also provide azlactone via direct addition to the external carbonate; this process would compete with the desired pseudo-intramolecular bond formation. Consequently, maintaining low substrate concentration is a prerequisite for controlling the reaction pathway and achieving high enantioselectivity.

(a)

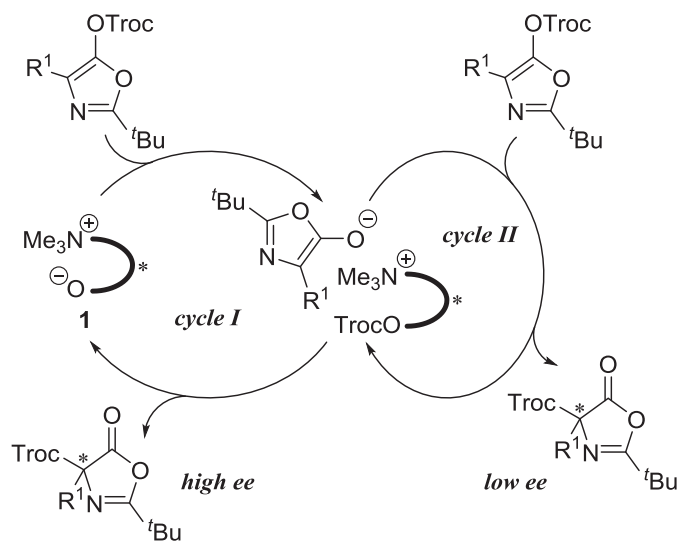

(b)

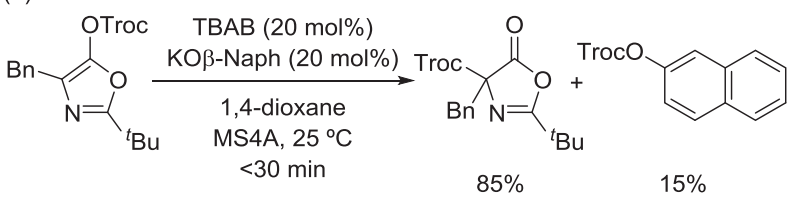

Figure 6. (a) Plausible reaction mechanisms of the chiral ammonium betaine 1-catalyzed Steglich rearrangement. (b) Intermolecular "Steglich rearrangement".

\subsection{Aldol Reaction of Oxindole-derived Enol Carbonates}

During the course of the study on the nucleophilic catalysis of ammonium betaines, we became interested in the moderate stability of the intermediary formed ammonium enolate, which eventually reacts with the aryl carbonate moiety pseudo-intramolecularly to give the "rearranged" product (Figure 7, path A). The possible intervention of the competing intermolecular reaction with the coexisting carbonate suggests that the transient enolate ion could be trapped by an external electrophile such as aldehyde, followed by $O$-acylation of the resulting alkoxylate, giving rise to a fully protected aldol adduct (path B) ${ }^{28}$ To examine this possibility, the aldol reaction of oxindole-derived vinylic carbonates with aldehydes was selected as a model reaction. Despite the oxindole core being commonly found in biologically relevant molecules, the catalytic asymmetric aldol reaction of oxindoles is scarcely developed and essentially limited to systems using highly activated aldehydes as requisite electrophiles. ${ }^{14,29}$ This is probably because aldol 
adducts of oxindoles tend to racemize under basic conditions via the retro-aldol reaction. ${ }^{30}$ With this respect, our approach would be generically advantageous because of the concurrent in situ acyl transfer to afford the aldol adduct as a stable, protected form.

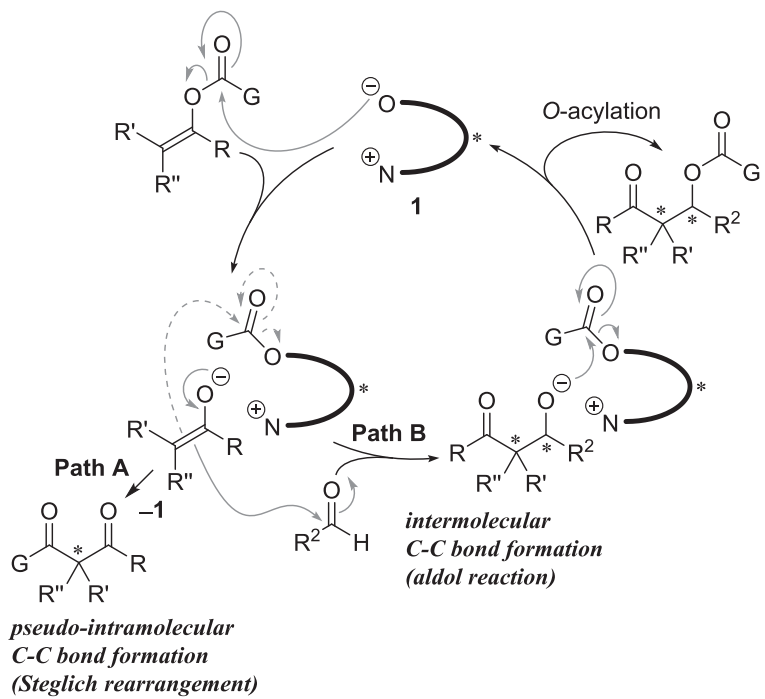

Figure 7. Working hypothesis for the chiral ammonium betaine 1-catalyzed aldol reaction.

The presumed aldol reaction of $O-2,2,2$-trichloroethoxycarbonyl enolate and benzaldehyde ( 2.0 equiv) was attempted with chiral ammonium betaine $\mathbf{1 j}$ as a nucleophilic catalyst. ${ }^{31}$ Fortunately, the expected adduct was obtained as a diastereomeric mixture ( $\mathrm{dr}=6: 1)$ in $77 \%$ yield with $34 \%$ ee for the major diastereomer, albeit notable amount of Steglich product was also isolated (aldol vs. Steglich=8:1) (Table 2, entry 1). ${ }^{32}$ The structural modification of $\mathbf{1}$ had a clear impact on the distribution of the products as well as stereoselectivity. Concretely, replacing the $N$-methyl substituent with sterically demanding groups dramatically improved selectivities, and 10 possessing a 3,5-bis(tert-butyldimethylsilyl)benzyl group enabled a promising level of control of both reaction pathway and stereochemical outcome (entries 3 and 4). Increasing the steric bulkiness of the aromatic substituent on the naphthyl unit (1p) further enhanced selectivities (entry 5). Because the parent structure of $\mathbf{1}$ is converted to $O$-acyl ammonium enolate upon the acyl-transfer reaction, the carbon-carbon bond-forming process is controlled by the $O$-acyl ammonium ion. Therefore, we investigated effect of the structure of $R^{1}$ in the substrate on selectivity profile (entries 6-8). When the trichloroethyl moiety was exchanged with a benzyl group, enantioselectivity was enhanced to $92 \%$, but the turnover frequency was markedly diminished (entry 6). This problem was overcome by introducing electron-withdrawing groups to the benzyl moiety, which improved reaction efficiency without affecting selectivities (entries 7 and 8). Eventually, use of the 3,5-bis(trifluoromethyl)benzyl carbonate in combination with $\mathbf{1 p}$ as a catalyst led to virtually complete discrimination of the reaction pathway with excellent stereocontrol (entry 8). Importantly, the reaction with DMAP as a representative nucleophilic catalyst under otherwise identical conditions exclusively gave the Steglich product $(12 \mathrm{~h}, 96 \%)$, illustrating the salient feature of the ionic nucleophilic catalysis of chiral ammonium betaine $\mathbf{1}$.

This unique asymmetric aldol protocol accommodated a
Table 2. Optimization of conditions for the chiral ammonium betaines 1-catalyzed aldol reaction.
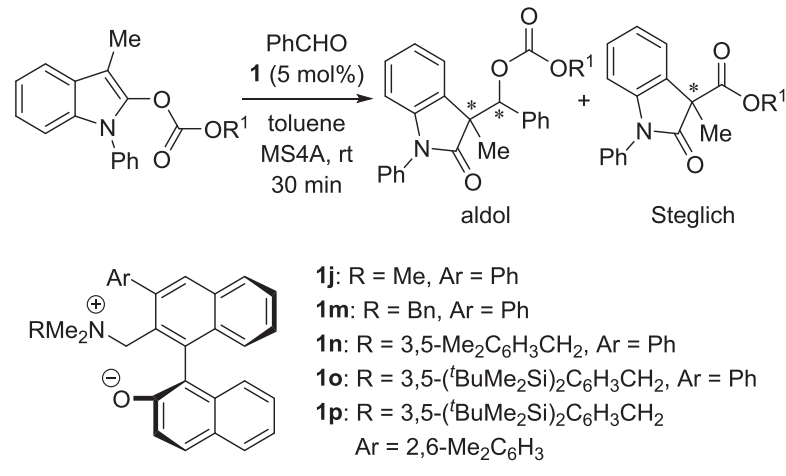

\begin{tabular}{ccccccc}
\hline entry & $\mathbf{1}$ & $\mathrm{R}^{1}$ & $\begin{array}{c}\text { yield } \\
(\%)\end{array}$ & $\mathrm{dr}$ & $\begin{array}{c}\text { aldol/ } \\
\text { Steglich }\end{array}$ & $\begin{array}{c}\text { ee } \\
(\%)\end{array}$ \\
\hline 1 & $\mathbf{1 j}$ & $\mathrm{Cl}_{3} \mathrm{CCH}_{2}$ & 77 & $6: 1$ & $8: 1$ & 34 \\
2 & $\mathbf{1} \mathbf{m}$ & $\mathrm{Cl}_{3} \mathrm{CCH}_{2}$ & 67 & $7: 1$ & $4: 1$ & 39 \\
3 & $\mathbf{1 n}$ & $\mathrm{Cl}_{3} \mathrm{CCH}_{2}$ & 79 & $15: 1$ & $15: 1$ & 71 \\
4 & $\mathbf{1 0}$ & $\mathrm{Cl}_{3} \mathrm{CCH}_{2}$ & 82 & $15: 1$ & $11: 1$ & 78 \\
5 & $\mathbf{1 p}$ & $\mathrm{Cl}_{3} \mathrm{CCH}_{2}$ & 86 & $\geq 20: 1$ & $13: 1$ & 89 \\
$6^{a}$ & $\mathbf{1 p}$ & $\mathrm{PhCH}_{2}$ & 51 & $\geq 20: 1$ & $\geq 20: 1$ & 92 \\
7 & $\mathbf{1 p}$ & $4-\mathrm{CF}_{3} \mathrm{C}_{6} \mathrm{H}_{4} \mathrm{CH}_{2}$ & 88 & $\geq 20: 1$ & $\geq 20: 1$ & 93 \\
8 & $\mathbf{1 p}$ & $3,5-\left(\mathrm{CF}_{3}\right)_{2} \mathrm{C}_{6} \mathrm{H}_{3} \mathrm{CH}_{2}$ & 89 & $\geq 20: 1$ & $\geq 20: 1$ & 95 \\
\hline${ }^{a}$ Reaction time was $12 \mathrm{~h} .^{2}$ & & & &
\end{tabular}

series of aromatic and aliphatic aldehydes, and high diastereoselectivity and excellent enantioselectivity were generally observed (Scheme 11). Moreover, various substituted oxindole-derived carbonates were also employable as the nucleophilic component. Noteworthy was that less than $5 \%$ of the corresponding Steglich product was detected in all cases.

Scheme 11. Chiral ammonium betaine 1p-catalyzed stereoselective aldol reaction.

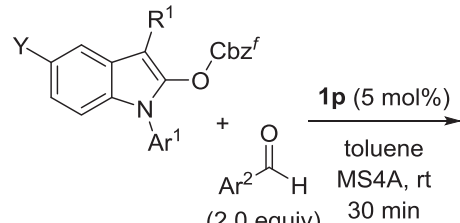

(2.0 equiv) $30 \mathrm{~min}$

$\mathrm{Cbz}^{f}=3,5-\left(\mathrm{CF}_{3}\right)_{2} \mathrm{C}_{6} \mathrm{H}_{3} \mathrm{CH}_{2} \mathrm{OCO}$

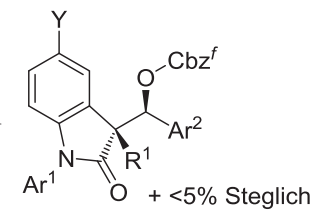

73 96\%, dr = 10 > 20:1 90 96\% ee

\section{Acridinium Betaine}

\subsection{PCET Catalysis}

As described, embedding an aryloxylate unit to a chiral ammonium ion has enabled ion-pair catalysis effective for achieving otherwise difficult highly stereoselective organic transformations. The judicious combination of functions of the aryloxylate, such as nucleophilicity, basicity, and the hydrogen-bonding capability of the conjugate acid, with the stereocontrolling ability of the ammonium ion allowed us to generate and control unique yet reactive ionic species. Based on these findings, we next turned our attention to another function of cationic species to further expand the potential of ion-pair catalysis of the betaine molecules by changing the structure of the cation component.

As an initial step toward this direction, we selected 9-mesityl acridinium ion, which was discovered by Fukuzumi in 2004 to be a redox active molecule having extremely long excited- 
state lifetime, ${ }^{33,34}$ as a functional cationic subunit and designed an intramolecular ion pair of type $\mathbf{3}$, acridinium betaine, as a single-electron-transfer catalyst (Figure 8). ${ }^{35}$ We hypothesized that the combination of the basic character of the aryloxylate moiety of $\mathbf{3}$ and the relatively high reduction potential of the acridinium ion at the ground state could facilitate protoncoupled electron transfer (PCET) in its hydrogen-bonding complex with an acidic substrate. Moreover, the resulting radical species could be utilized for downstream bond formation. PCET is one of the basic mechanisms of chemical and biological transformations, where simultaneous transfer of one electron and one proton between given substrates generates highenergy radical intermediates. ${ }^{36}$ Essentially, PCET is known to proceed via a more stable transition state than that expected in iterative electron-proton (or proton-electron) transfer processes. Therefore, in order to catalytically promote the PCET reaction, the reduction/oxidation potential of the catalyst is expected to be much lower than that of the two-step reaction, and the resulting reduced (or oxidized) catalyst should be restored under accessible conditions. Although fulfilling these requirements could lead to the development a catalytically operative chemical redox reagent, this possibility has not yet been experimentally explored.

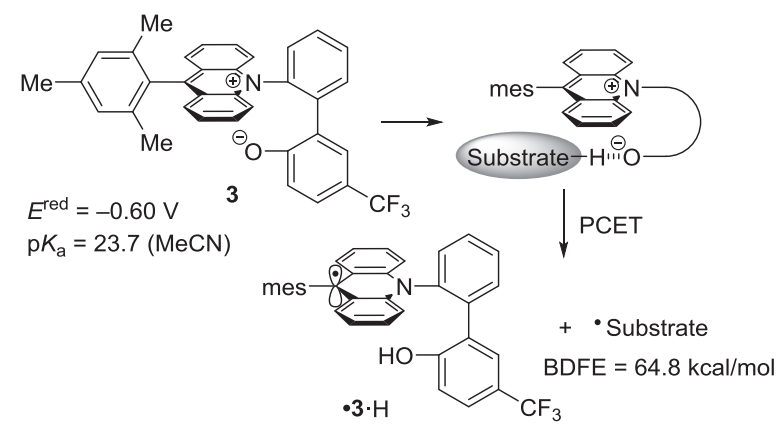

Figure 8. Structure and properties of acridinium betaine $\mathbf{3}$ and working hypothesis for generating a radical species via the PCET process.

The single-electron transfer reaction by chemical redox reagents is a powerful means for generating radical species, while it generally requires an over-stoichiometric amount of strong reductants (or oxidants), such as low-valent metal salts, which produce non-reusable waste after the reaction. ${ }^{37}$ Largely because external energy infusion is not necessary, the chemical redox reaction is highly reliable and it is still frequently used for the synthesis of complex molecules. Therefore, the advent of new catalysts or catalytic systems of chemical redox reactions using a clean terminal oxidant such as oxygen is in high demand from the viewpoint of sustainable process development. However, the exploration of catalytically relevant chemical redox reagents has been scarce, presumably because of difficulties associated with regeneration of the catalyst. Generation of reactive radical species from stable compounds requires high-energy redox-active reagents, which would make it difficult to regenerate the catalyst by commonly utilized terminal oxidants/reductants. We anticipated that a catalytic PCET reaction based on the use of $\mathbf{3}$ could offer a platform for overcoming this dilemma because the acridinium ion is known to be robust to the single-electron redox process and is regenerated via autoxidation of its reduced radical form. ${ }^{34}$

Acridinium betaine $\mathbf{3}$ was synthesized as a stable solid in a straightforward manner and its physical properties were analyzed. $^{34}$ The reduction potential of $\mathbf{3}$ was determined to be $-0.60 \mathrm{~V}$ (vs. SCE) by cyclic voltammetry (CV) in $\mathrm{MeCN}$ and its $\mathrm{p} K_{\mathrm{a}}(23.7$ in $\mathrm{MeCN})$ value was derived from the change in UV-Vis absorbance during acid-base titration with $3 \cdot \mathrm{HCl} /$ DBU or $\mathbf{3} /$ acetic acid. According to these physical data, 3 can oxidize organic compounds containing an active proton (bond-dissociation free energy $($ BDFE) $=64.8 \mathrm{kcal} / \mathrm{mol}$ ) through the PCET process. As a model reaction for evaluating the catalytic property of $\mathbf{3}$, we selected homodimerization of $N$-PMP-3-aryl-oxindole $(\mathrm{BDFE}=64.8 \mathrm{kcal} / \mathrm{mol}$, calculated at $\mathrm{PCM}(\mathrm{MeCN})-\mathrm{UB} 3 \mathrm{LYP} / 6-311++\mathrm{G}(2 \mathrm{df}, 2 \mathrm{p})$ level $)^{38}$ via a radical enolate.

The viability of our hypothesis was initially investigated by treatment of $N$-PMP-3-phenyl-oxindole with 3 ( $2 \mathrm{~mol} \%)$ in toluene at room temperature under oxygen atmosphere (Table 3, entry 1). The reaction proceeded smoothly and the expected dimer was isolated in good yield after $3 \mathrm{~h}$ of stirring. While a similar result was obtained under atmospheric conditions, the reaction was completely suppressed under argon atmosphere (entries 2 and 3). This observation clearly indicates that oxygen is essential for the reaction. The critical importance of the structure of $\mathbf{3}$, with respect to both the redoxactive unit and the basic site embedded in the same molecular framework, was confirmed by the following reactions (entries 4-6). A simple base catalyst, pyridinium betaine (betaine), did not afford the homodimerized product under similar conditions (entry 4). On the other hand, acridinium salt (acr) subtly promoted the coupling reaction and certain acceleration was observed under binary catalysis of acr and betaine; however, the rates were markedly slower than that in the 3-catalyzed reaction (entries 5 and 6). The relative configuration of the dimer was unambiguously determined by $\mathrm{X}$-ray diffraction analysis to be meso-isomer. ${ }^{39}$

Table 3. Reaction conditions.
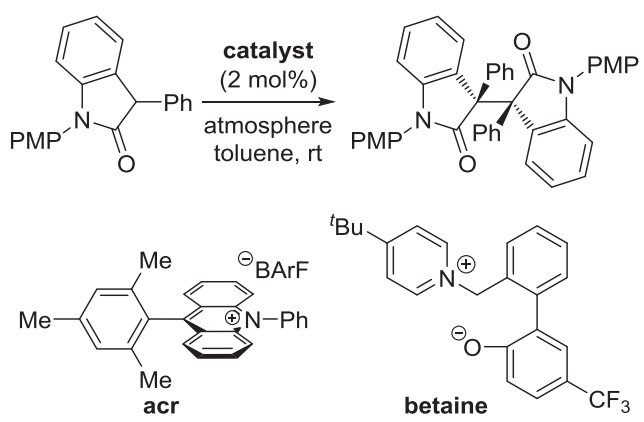

\begin{tabular}{ccccc}
\hline entry & catalyst & atmosphere & time $(\mathrm{h})$ & yield (\%) \\
\hline 1 & 3 & $\mathrm{O}_{2}$ & 3 & 80 \\
2 & 3 & air & 3 & 82 \\
3 & 3 & $\mathrm{Ar}$ & 24 & $<5$ \\
4 & betaine & air & 24 & trace \\
5 & acr & air & 24 & 6 \\
6 & betaine + acr & air & 3 & 29 \\
\hline
\end{tabular}

To gain a mechanistic insight into the 3-catalyzed dimerization of oxindole, the kinetic profile was analyzed by monitoring the reaction progress with in situ IR measurement. Comparison of the initial rate between the 3-catalyzed reaction and that under the binary catalysis of betaine and acr revealed that the intramolecular ion-pair structure of $\mathbf{3}$ was 
crucial for enhancing catalytic efficiency. The order of the reaction on each component was then determined as: $1^{\text {st }}$ order on $1,-0.85^{\text {th }}$ order on oxindole, and $0.5^{\text {th }}$ order on $\mathrm{O}_{2}$. The observed non-integer orders suggest the intervention of equilibrium in the rate-limiting step, and a possible mechanism is shown in Figure 9. The negative dependence on oxindole concentration could be accounted for by that SET from the oxindole enolate to the acridinium unit would be much more sluggish than PCET, and generation of the acridinium enolate may impede regeneration of $\mathbf{3}$, where the effective concentration of 3 was decreased in the presence of excess amount of the oxindole. The role of $\mathrm{O}_{2}$ as the single-electron oxidant for liberating the radical enolate of oxindole and formation of $\mathrm{H}_{2} \mathrm{O}_{2}$ was experimentally proved, providing further evidence for the proposed reaction mechanism. ${ }^{40}$ Although additional study is still required to clarify the detailed mechanism, the above observations support the PCET catalysis of $\mathbf{3}$ depicted in Figure 9.

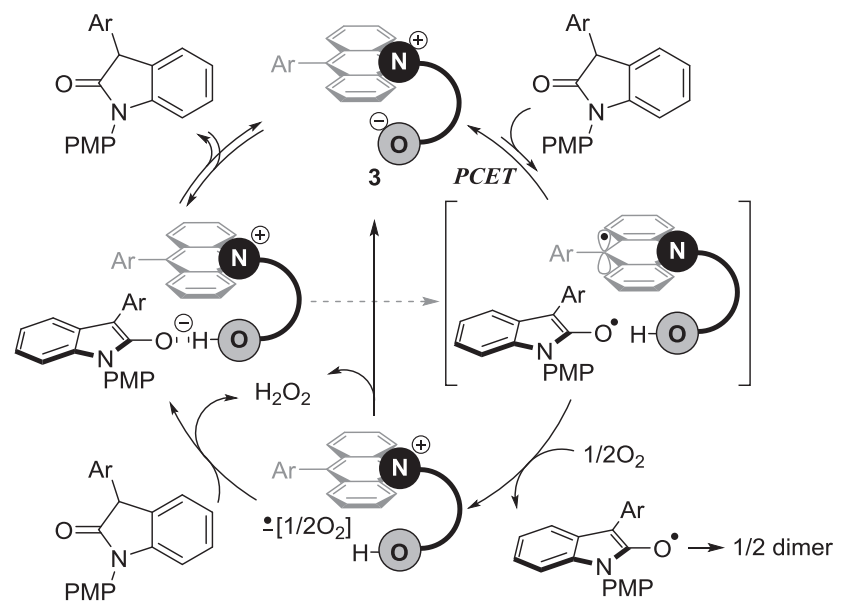

Figure 9. Proposed reaction mechanism for the acridinium betaine-catalyzed homodimerization of 3-aryl oxindoles via the PCET process.

\section{Conclusion}

As a unique means for realizing a cooperative catalysis of a cation and anion, an ion-pair catalysis, we developed an ammonium betaine of type $\mathbf{1}$ as an intramolecular ion-pair catalyst and unveiled its catalytic performance in achieving a series of selective organic transformations. With particular focus on the functions of the anionic moiety, an aryloxylate, both bifunctional base catalysis and ionic nucleophilic catalysis were established in the development of highly stereoselective bond-forming reactions. Upon giving an eye to the function of the cationic moiety, the organic chemical-redox catalyst 3 was elaborated by employing the acridinium ion as a requisite cationic unit having single-electron redox capability. The nature of these catalyses is distinct from ordinary bifunctional onium salt catalysis and represents a true ion-pair catalysis. ${ }^{41}$ Our studies on the chemistry of betaines demonstrate the potential of the catalysis that relies on imparting an appropriate yet discrete structure to an ion pair in selective organic synthesis and will stimulate further research efforts for understanding and exploitation of ion-pair catalysis. ${ }^{42}$

\section{Acknowledgements}

The authors would like to express their sincere apprecia- tion to all past and present members of the betaine team in the laboratory for the chemistry of organic reaction (Ooi group) at Nagoya University for their vital contributions. Financial support was provided by CREST-JST (JPMJCR13L2: 13418441), a Grant-in-Aid for Scientific Research on Innovative Areas "Advanced Molecular Transformations by Organocatalysts" from MEXT, the Program for Leading Graduate Schools "Integrative Graduate Education and Research Program in Green Natural Sciences" in Nagoya University, and Grants of JSPS for Scientific Research.

\section{References}

1) Dolling, U.-H.; Davis, P.; Grabowski, E. J. J. J. Am. Chem. Soc. 1984 $106,446$.

2) Brak, K.; Jacobsen, E. N. Angew. Chem. Int. Ed. 2013, 52, 534.

3) (a) Jones, R. A. in Quaternary Ammonium Salts: Their use in PhaseTransfer Catalysis; Academic Press: London, U.K., 2001. (b) Asymmetric Phase Transfer Catalysis; Maruoka, K., Ed.; Wiley-VCH Weinheim, Germany, 2008. (c) Ooi, T.; Maruoka, K. Angew. Chem Int. Ed. 2007, 46, 4222. (d) Shirakawa, S.; Maruoka, K. Angew. Chem. Int. Ed. 2013, 52, 4312. (d) Tan, J.; Yasuda, N. Org. Process Res. Dev. 2015, 19, 1731

4) Brière, J.-F.; Oudeyer, S.; Dalla, V.; Levacher, V. Chem. Soc. Rev. 2012, 41,1696 .

5) Day, C. R.; Kempson, S. A. Biochim. Biophys. Acta 2016, 1860, 1098.

6) Godemert, J.; Oudeyer, S.; Levacher, V. Chem CatChem 2016, 8, 74.

7) (a) Uraguchi, D.; Koshimoto, K.; Ooi, T. J. Am. Chem. Soc. 2008, 130, 10878. (b) Uraguchi, D.; Koshimoto, K.; Sanada, C.; Ooi, T. Tetrahedron: Asymmetry 2010, 21, 1189.

8) Uraguchi, D.; Koshimoto, K.; Ooi, T. Chem. Commun. 2010, 46, 300.

9) When an anionic component of the betaine precursor was trifluoroacetate $\mathrm{CF}_{3} \mathrm{CO}_{2}{ }^{-}$, complete conversion to the corresponding betaine 1 could be confirmed by disappearance of the signal of $\mathrm{CF}_{3} \mathrm{CO}_{2}{ }^{-}$in ${ }^{19} \mathrm{~F}$ NMR analysis.

10) Catalytic asymmetric synthesis of $\alpha$-substituted $\alpha, \beta$-diamino acids: (a) Knudsen, K. R.; Jørgensen, K. A. Org. Biomol. Chem. 2005, 3 , 1362. (b) Chen, Z.; Morimoto, H.; Matsunaga, S.; Shibasaki, M. J. Am. Chem. Soc. 2008, 130, 2170. (c) Singh, A.; Johnston, J. N. J. Am Chem. Soc. 2008, 130, 5866. (d) Uraguchi, D.; Ueki, Y.; Ooi, T. J. Am. Chem. Soc. 2008, 130, 14088. (e) Hernández-Toribio, J.; Arrayás, R. G.; Carretero, J. C. J. Am. Chem. Soc. 2008, 130, 16150. (f) Shi, S.-H. Huang, F.-P.; Zhu, P.; Dong, Z.-W.; Hui, X.-P. Org. Lett. 2012, 14, 2010. (g) Sun, Z.; Weidner, K.; Kumagai, N.; Shibasaki, M. Chem Eur. J. 2015, 21, 17574. (h) Kikuchi, J.; Momiyama, N.; Terada, M. Org. Lett. 2016, 18, 2521. (i) Sprague, D. J.; Singh, A.; Johnston, J. N. Chem. Sci. 2018, 9, 2336. See also: (j) Arrayás, R. G.; Carretero, J. C. Chem. Soc. Rev. 2009, 38, 1940.

11) (a) Lin, Y.; Andersen, K. K. Eur. J. Org. Chem. 2002, 557. (b) Uraguchi, D.; Yamada, K.; Ooi, T. Angew. Chem. Int. Ed. 2015, 54, 9954.

12) (a) Mosey, R. A.; Fisk, J. S.; Tepe, J. J. Tetrahedron: Asymmetry 2008, 19, 2755. (b) Alba, A.-N. R.; Rios, R. Chem. Asian J. 2011, 6, 720. (c) de Castro, P. P.; Carpanez, A. G.; Amarante, G. W. Chem. Eur. J. 2016, 22, 10294.

13) Torii, M.; Kato, K.; Uraguchi, D.; Ooi, T. Beilstein J. Org. Chem. 2016, 12, 2099

14) (a) Marti, C.; Carreira, E. M. Eur. J. Org. Chem. 2003, 2209. (b) Galliford, C. V.; Scheidt, K. A. Angew. Chem. Int. Ed. 2007, 46, 8748 (c) Trost, B. M.; Brennan, M. K. Synthesis 2009, 3003. (d) Singh, G. S.; Desta, Z. Y. Chem. Rev. 2012, 112, 6104. (e) Zhou, F.; Liu, Y.-L.; Zhou, J. Adv. Synth. Catal. 2010, 352, 1381. (f) Dalpozzo, R.; Bartoli, G.; Bencivenni, G. Chem. Soc. Rev. 2012, 41, 7247. (g) Ziarani, G. M.; Gholamzadeh, P.; Lashgari, N.; Hajiabbasi, P. ARKIVOC 2013, (i), 470. (h) Cao, Z.-Y.; Wang, Y.-H.; Zeng, X.-P.; Zhou, J. Tetrahedron Lett. 2014, 55, 2571 .

15) (a) Tian, X.; Jiang, K.; Peng, J.; Du, W.; Chen, Y.-C. Org. Lett. 2008 10, 3583. (b) Cheng, L.; Liu, L.; Jia, H.; Wang, D.; Chen, Y.-J. J. Org. Chem. 2009, 74, 4650. (c) He, R.; Ding, C.; Maruoka, K. Angew. Chem. Int. Ed. 2009, 48, 4559. (d) Jin, Y.; Chen, D.; Zhang, X. R. Chirality 2014, 26, 801. (e) Shimizu, S.; Tsubogo, T.; Xu, P.; Kobayashi, S. Org. Lett. 2015, 17, 2006.

16) Uraguchi, D.; Torii, M.; Kato, K.; Ooi, T. Heterocycles 2017, 94, 441.

17) Sridharan, V.; Suryavanshi, P. A.; Menéndez, J. C. Chem. Rev. 2011, 111,7157 
18) (a) Ono, N. in The Nitro Group in Organic Synthesis, Wiley-VCH: New York, 2001. (b) Marqués-López, E.; Merino, P.; Tejero, T.; Herrera, R. P. Eur. J. Org. Chem. 2009, 2401. (c) Noble, A.; Anderson, J. C. Chem. Rev. 2013, 113, 2887.

19) (a) Casiraghi, G.; Zanardi, F.; Appendino, G.; Rassu, G. Chem. Rev. 2000, 100, 1929. (b) Kalesse, M. Top. Curr. Chem. 2005, 244, 43

20) Uraguchi, D.; Oyaizu, K.; Ooi, T. Chem. Eur. J. 2012, 18, 8306.

21) Oyaizu, K.; Uraguchi, D.; Ooi, T. Chem. Commun. 2015, 51, 4437.

22) Uraguchi, D.; Oyaizu, K.; Noguchi, H.; Ooi, T. Chem. Asian J. 2015, 10, 334

23) (a) Davis, T. A.; Johnston, J. N. Chem. Sci. 2011, 2, 1076. (b) Davis, T. A.; Vilgelm, A. E.; Richmond, A.; Johnston, J. N. J. Org. Chem. 2013, 78, 10605. (c) Vara, B. A.; Mayasundari, A.; Tellis, J. C.; Danneman, M. W.; Arredondo, V.; Davis, T. A.; Min, J.; Finch, K.; Guy, R. K.; Johnston, J. N. J. Org. Chem. 2014, 79, 6913.

24) (a) Wurz, R. P. Chem. Rev. 2007, 107, 5570. (b) Enders, D.; Niemeier, O.; Henseler, A. Chem. Rev. 2007, 107, 5606. (c) Marion, N.; Díez-González, S.; Nolan, S. P. Angew. Chem. Int. Ed. 2007, 46, 2988. (d) Denmark, S. E.; Beutner, G. L. Angew. Chem. Int. Ed. 2008, 47, 1560.

25) For a review, see: (a) Moyano, A.; El-Hamdouni, N.; Atlamsani, A. Chem. Eur. J. 2010, 16, 5260. See recent examples: (b) Mandai, H.; Fujii, K.; Yasuhara, H.; Abe, K.; Mitsudo, K.; Korenaga, T.; Suga, S. Nat. Commun. 2016, 7, 11297. (c) Yamamoto, T.; Murakami, R.; Suginome, M. J. Am. Chem. Soc. 2017, 139, 2557. (d) Cruchter, T.; Medvedev, M. G.; Shen, X.; Mietke, T.; Harms, K.; Marsch, M.; Meggers, E. ACS Catal. 2017, 7, 5151.

26) Uraguchi, D.; Koshimoto, K.; Miyake, S.; Ooi, T. Angew. Chem. Int. Ed. 2010, 49, 5567.

27) When a betaine lacking 3-substituent of the aryloxylate side was prepared, a methanolic solution of its conjugate acid salt was passed through a column of ion-exchange resin ( $\mathrm{OH}^{-}$form).

28) (a) Ryan, S. J.; Candish, L.; Lupton, D. W. J. Am. Chem. Soc. 2009, 131, 14176. (b) Candish, L.; Lupton, D. W. Org. Lett. 2010, 12, 4836. (c) De, C. K.; Mittal, N.; Seidel, D. J. Am. Chem. Soc. 2011, 133, 16802. (d) Candish, L.; Lupton, D. W. Chem. Sci. 2012, 3, 380.

29) (a) Ogawa, S.; Shibata, N.; Inagaki, J.; Nakamura, S.; Toru, T.; Shiro, M. Angew. Chem. Int. Ed. 2007, 46, 8666. (b) Shen, K.; Liu, X.; Zheng, K.; Li, W.; Hu, X.; Lin, L.; Feng, X. Chem. Eur. J. 2010, 16, 3736. (c) Shen, K.; Liu, X.; Wang, W.; Wang, G.; Cao, W.; Li, W.; Hu, X.; Lin, L.; Feng, X. Chem. Sci. 2010, 1, 590.

30) (a) Pesciaioli, F.; Righi, P.; Mazzanti, A.; Gianelli, C.; Mancinelli, M.; Bartoli, G.; Bencivenni, G. Adv. Synth. Catal. 2011, 353, 2953. See also: (b) Chen, W.-B.; Wu, Z.-J.; Hu, J.; Cun, L.-F.; Zhang, X.-M.; Yuan, W.-C. Org. Lett. 2011, 13, 2472.

31) Uraguchi, D.; Koshimoto, K.; Ooi, T. J. Am. Chem. Soc. 2012, 134, 6972.

32) The enantiomeric excess of the Steglich rearrangement product was not analyzed

33) Fukuzumi, S.; Kotani, H.; Ohkubo, K.; Ogo, S.; Tkachenko, N. V.; Lemmetyinen, H. J. Am. Chem. Soc. 2004, 126, 1600.

34) Wilger, D. J.; Grandjean, J.-M. M.; Lammert, T. R.; Nicewicz, D. A. Nat. Chem. 2014, 6, 720.

35) Uraguchi, D.; Torii, M.; Ooi, T. ACS Catal. 2017, 7, 2765.

36) For reviews on PCET, see: (a) Huynh, M. H. V.; Meyer, T. J. Chem. Rev. 2007, 107, 5004. (b) Weinberg, D. R.; Gagliardi, C. J.; Hull, J. F.; Murphy, C. F.; Kent, C. A.; Westlake, B. C.; Paul, A.; Ess, D. H.; McCafferty, D. G.; Meyer, T. J. Chem. Rev. 2012, 112, 4016. (c) Yayla, H. G.; Knowles, R. R. Synlett 2014, 25, 2819. (d) Gentry, E. C.; Knowles, R. R. Acc. Chem. Res. 2016, 49, 1546. See also: (e) Miller, D. C.; Tarantino, K. T.; Knowles, R. R. Top. Curr. Chem. 2016, 374, 30.

37) (a) Rosen, B. M.; Percec, V. Chem. Rev. 2009, 109, 5069. (b) Szostak, M.; Spain, M.; Procter, D. J. Chem. Soc. Rev. 2013, 42, 9155. (c) Zhang, N.; Samanta, S. R.; Rosen, B. M.; Percec, V. Chem. Rev. 2014, 114, 5848. (d) Jia, X. Synthesis 2016, 48, 18. (e) Tellis, J. C.; Kelly, C. B.; Primer, D. N.; Jouffroy, M.; Patel, N. R.; Molander, G. A. Acc. Chem. Res. 2016, 49, 1429.

38) Gaussian 09, Revision D. 01., Frisch, M. J. et al. Gaussian, Inc.: Wallingford, CT, 2013.

39) The reason for the observed diastereopreference is unclear yet.

40) Matsubara, C.; Kawamoto, N.; Takamura, K. Analyst 1992, 117, 1781

41) Novacek, J.; Waser, M. Eur. J. Org. Chem. 2013, 637.

42) (a) Zhang, W.-Q.; Cheng, L.-F.; Yu, J.; Gong, L.-Z. Angew. Chem. Int. Ed. 2012, 51, 4085. (b) Claraz, A.; Landelle, G.; Oudeyer, S.; Levacher, V. Eur. J. Org. Chem. 2013, 7693. (c) Zhou, X.; Wu, Y.; Deng, L. J. Am. Chem. Soc. 2016, 138, 12297.

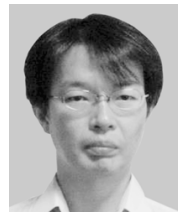

Daisuke Uraguchi is an Associate Professor of Nagoya University. He was born in 1974 in Hokkaido and received his B.Sc. (1997) and Ph.D. degree (2002) from Hokkaido University under the guidance of Professor Keiji Maruoka. He gained substantial experience working as a postdoctoral fellow of JSPS (2002-2004) with Professor P. Andrew Evans at Indiana University and with Professor Masahiro Terada at Tohoku University. Then, he moved to the Sagami Chemical Research Center (2004-2006). In 2006, he was appointed as Assistant Professor of the research group of Professor Takashi Ooi at Nagoya University, and promoted to Associate Professor in 2008. He has received the Takeda Pharmaceutical Co., Ltd. Award in SSOCJ (2008), The CSJ Award for Young Chemist (2010), The Young Scientists' Prize from MEXT (2011), Banyu Chemist Award (2012), Lectureship Award MBLA (2013), and Thieme Chemistry Journal Award (2014). His current research interests include the chemistry of organic ion-pair catalysis.

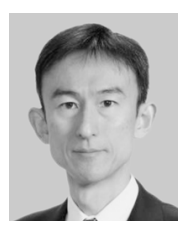

Takashi Ooi is a Professor of Nagoya University. He was born in 1965 in Nagoya and received his B.Eng. (1989) and Ph.D. (1994) from Nagoya University under the supervision of Professor Hisashi Yamamoto. After working as a postdoctoral fellow of JSPS with Professor Julius Rebek, Jr. at Massachusetts Institute of Technology, he started his academic career as an Assistant Professor of Professor Keiji Maruoka's group at Hokkaido University in 1995. In 2001, he was appointed as an Associate Professor at Kyoto University. He was then promoted to Professor of Nagoya University in 2006. From 2012, he has been one of the principal investigators at the Institute of Transformative Bio-Molecules in Nagoya University (WPI-ITbM). He has received Chugai Pharmaceutical Co., Ltd. Award in SSOCJ (1996), the CSJ Award for Young Chemists (1999), Thieme Journal Award (2006), JSPS prize (2010), IBM Japan Science Prize (2011), Inoue Prize for Science (2013), Fellow of the RSC (FRSC) (2014), and SSOCJ DaiichiSankyo Award for Medicinal Organic Chemistry (2017). His research interests are general synthetic organic chemistry. 